\title{
A comparison of the modified Broström procedure and modified Karlsson procedure in treating chronic lateral ankle instability: a systematic review and meta-analysis
}

\author{
Xinchao Deng ${ }^{1}$, Man Zou ${ }^{2}$, Hongfei Zhu ${ }^{3}$, Changjun Zuo ${ }^{4}$, Kunqing Li ${ }^{5}$, Liang Qian ${ }^{1}$ \\ ${ }^{1}$ Department of Orthopedics, Wuhan Eighth Hospital, Wuhan, China; ${ }^{2}$ Respiratory Department, Wuhan Eighth Hospital, Wuhan, China; \\ ${ }^{3}$ Anesthesiology Department, Hubei Hospital of Traditional Chinese Medicine, Hubei Academy of Traditional Chinese Medicine, Wuhan, China; \\ ${ }^{4}$ Department of Orthopedics, Hospital of Hubei 672 Integrated Traditional Chinese and Western Medicine, Wuhan, China; ${ }^{5}$ Department of \\ Orthopaedics, General Hospital of Hubei Jianghan Oilfield Affiliated with Yangtze University, Qianjiang, China \\ Contributions: (I) Conception and design: X Deng, L Qian; (II) Administrative support: M Zou; (III) Provision of study materials or patients: H Zhu; \\ (IV) Collection and assembly of data: C Zuo; (V) Data analysis and interpretation: K Li; (VI) Manuscript writing: All authors; (VII) Final approval of \\ manuscript: All authors. \\ Correspondence to: Liang Qian. Department of Orthopedics, Wuhan Eighth Hospital, Wuhan 430010, China. Email: dtrhnz@163.com.
}

\begin{abstract}
Background: This study sought to compare the efficacy and surgery complications of the modified Broström procedure and the modified Karlsson procedure in treating patients with chronic lateral ankle instability (CLAI).

Methods: Full-text publications on the clinical efficacy of Broström's and Karlsson's procedures were retrieved from multiple databases. Review Manager 5.0 was adopted for the meta-analysis, sensitivity analysis, and bias analysis.

Results: Nine studies comprising a total of 643 patients were identified. The meta-analysis suggested that the American Orthopedic Foot and Ankle Society (AOFAS) scores of patients in the Karlsson group were higher than those of patients in the Broström group [mean deviation $(\mathrm{MD})=6.31,95 \%$ confidence interval (CI): 2.31-10.30, $\mathrm{P}=0.002$; P for heterogeneity $<0.00001, \mathrm{I}^{2}=58 \%$. The Tegner scores of patients in the Karlsson group were higher than those of patients in the Broström group (MD =0.72, 95\% CI: 0.48-0.95, P=0.24; P for heterogeneity $\left.<0.00001, \mathrm{I}^{2}=23 \%\right)$. Operation times in the Broström group were higher than those in the Karlsson group ( $\mathrm{MD}=-15.50,95 \% \mathrm{CI}$ : $-19.98--11.02, \mathrm{P}<0.00001$; $\mathrm{P}$ for heterogeneity $\left.<0.00001, \mathrm{I}^{2}=63 \%\right)$. Patients in the Karlsson group had higher levels of satisfaction than those in the Broström group ( $\mathrm{MD}=0.63$, 95\% CI: $0.47-0.79, \mathrm{P}<0.00001$; $\mathrm{P}$ for heterogeneity $=0.91, \mathrm{I}^{2}=0 \%$ ). No significant difference was observed in surgery complications between the Karlsson and Broström groups [odds ratio $(\mathrm{OR})=1.71,95 \% \mathrm{CI}: 0.79-3.71, \mathrm{P}=0.18$; $\mathrm{P}$ for heterogeneity $=0.99, \mathrm{I}^{2}=0 \%$ ].
\end{abstract}

Discussion: Based on the heterogeneity analysis results, this study showed that Karlsson's procedure was more efficient and safer than Broström's treatment in the treatment of CLAI patients.

Keywordsł Karlsson; Broström; chronic lateral ankle instability (CLAI); therapy

Submitted Mar 19, 2021. Accepted for publication Jun 17, 2021.

doi: 10.21037/apm-21-963

View this article at: https://dx.doi.org/10.21037/apm-21-963

\section{Introduction}

The incidence of all sport injuries is $16-21 \%$, and the ankle sprain is the most common sport injury (1). An ankle sprain causes the rupture of the anterior talofibular ligament and calcaneofibular ligament (1-3). If not treated promptly, chronic lateral ankle instability (CLAI) of the joint can occur, causing repeated sprain, instability, and chronic pain (2). The lateral collateral ligaments of the ankle joint 
primarily include the anterior talofibular ligament, the calcaneofibular ligament, and the posterior talofibular ligament. The first 2 ligaments form the core structure that maintain the lateral stability of the ankle joint by limiting the inversion and forward movement of the talus when the ankle joint flexes and extends dorsally (4-6). Injury of the lateral ankle ligament can lead to excessive varus and forward instability of the ankle joint. Any failure to promptly treat the injury could lead to repeated sprains of the joint and secondary intra-articular injuries, such as cartilage injuries. Previously, several methods of reconstruction have been shown to restore the lateral stability of the ankle joint. However, despite some satisfactory results being achieved, these methods have some drawbacks.

The modified Broström and Karlsson procedures are two repair operations. The modified Broström procedure is the main method used in repair operations to effectively tighten the tissue. The advantage of this procedure is that the trauma to the patient's body is relatively minimal; however, disadvantages of this procedure include some damage to the ligament body and the need to repair the stop point (7-9). Additionally, it is difficult to suture the remaining ligament tissue to the normal endpoint, and its stability cannot be guaranteed in a long term. Conversely, the modified Karlsson procedure alters the way in which the bone passage is made when reconstructing the original operation termination point, such that the suture anchor is used to suture and fix the broken end of the ligament. This reduces the time required to make the bone passage and shortens the operation time, thereby make the operation process convenient (10-12).

Several articles have compared these two methods in relation to various research designs, the inclusion and exclusion criteria, and the measurements. In this study, we conducted a meta-analysis to comprehensively evaluate the clinical efficacy and surgery complications of the modified Broström and Karlsson procedures. We present the following article in accordance with the PRISMA reporting checklist (available at https://dx.doi. org/10.21037/apm-21-963).

\section{Methods}

\section{Searched databases and strategies}

The PubMed, EMBASE, China National Knowledge Infrastructure, and the Central Register of Cochrane Controlled Trials databases were searched (without language restrictions) for items published after 2010 . Two review team members independently conducted the preliminary searches, deleted any duplicate records, screened the titles and abstracts for relevance, checked the reference lists of all publications of interest for references to other related publications, and determined whether the publications should be excluded or required further evaluation. Next, a review of the full text of each publication was undertaken to determine if the studies met the inclusion criteria. The full-text articles designated for inclusion were then reviewed, and the references of the retrieved articles and previous reviews were manually checked to identify any other eligible studies. The manual review of the reference lists of the retrieved articles and previous comments on the topics of interest enabled other relevant research to be collected.

\section{Inclusion and exclusion criteria}

Publications were included in the analysis if:

(I) They were randomized trials, retrospective studies, or case-control studies;

(II) They compared the modified Karlsson procedure and the modified Broström procedure;

(III) The study participants included patients with CLAI.

Conversely, publications were excluded from the analysis if:

(I) They were case studies, meta-analyses or letters to editors;

(II) No comparison was made between the modified Karlsson procedure and the modified Broström procedure;

(III) The study did not include patients without CLAI;

(IV) The publications were duplicates; and/or

(V) Only the abstracts for the publications were available.

\section{Data extraction and review}

After making the selection, the 2 authors analyzed the selected studies and identified basic information from the article, including details of the author(s), year of publication, study location, sample characteristic data (i.e., the number and age of patients in each study), and intervention type and analysis results. Then we extract the data. If any issue arose between the 2 reviewers, the issue was discussed and resolved with the assistance of a third reviewer. The risk 


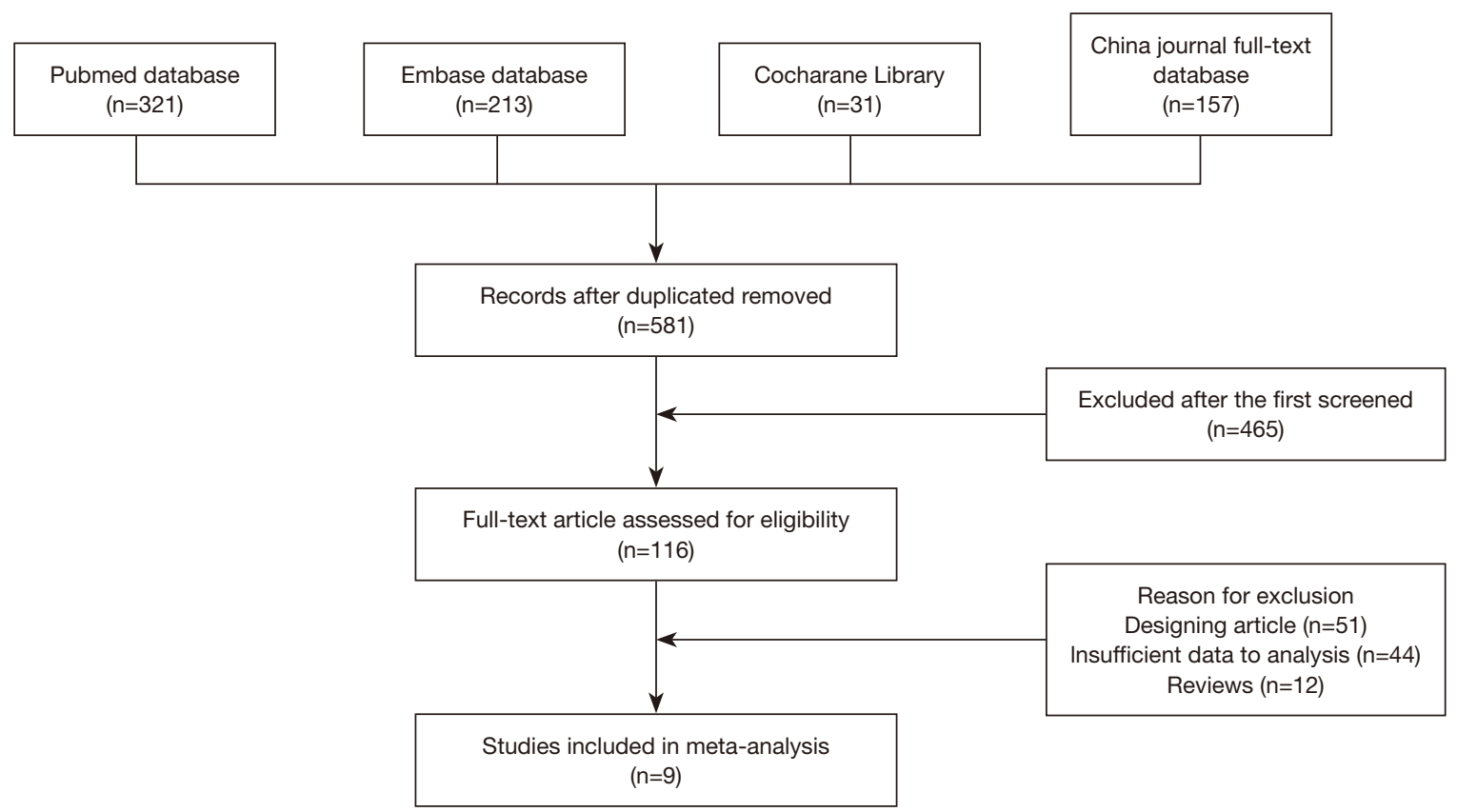

Figure 1 Schematic representation of the study identification process and the inclusion and exclusion criteria.

assessment of bias in the study was conducted using a risk assessment tool recommended by Cochrane Collaboration. The overall risk of bias assessment for each study was divided into green (a low risk of bias), yellow (an unclear risk of bias), and red (a high risk of bias) according to the standard.

\section{Statistical analysis}

The Cochran-Mantel-Haenszel method implemented in Review Manager (RevMan) version 5.0 (Copenhagen: Nordic Cochrane Center, Cochrane Collaboration, 2008) was used for the calculation and analysis. Combined odds ratios (ORs) and $95 \%$ confidence intervals (CIs) were evaluated. In relation to the continuous variables, the ratios of the mean \pm standard deviation of the continuous data and $95 \%$ CI were used. In this study, a $\mathrm{P}$ value of $<0.05$ was considered statistically significant. To evaluate the existence and degree of statistical heterogeneity, the Cochran Q-test and $\mathrm{I}^{2}$ index were used. If there was a $\mathrm{I}^{2}>50 \%$ and/or a $\mathrm{Q}$-test with a $\mathrm{P}$ value $<0.05$, which indicated large heterogeneity, the random-effects model was used; otherwise the fixedeffects model was used. In this study, a funnel chart was used to assess symmetry to detect the possibility of publication bias. In addition, a sensitivity analysis was performed according to the quality and weight of the test, and individual tests were excluded.

\section{Results}

\section{Search results}

In this study, a total of 581 articles were retrieved following a search of the electronic databases. After comprehensive reading, a total of 9 articles were found to meet the inclusion criteria for this study. The remaining 572 articles were excluded due to duplication, irrelevance, the lack of a control group, incomplete data or comparison, incomplete reviews, or only the articles being unavailable. Figure 1 shows a flowchart of the research process and the inclusion and exclusion criteria.

\section{Main characteristics of the studies}

Table 1 sets out the types of studies included in the metaanalysis and patient information for each group. The present meta-analysis comprised a total of 643 patients from 9 randomized trials $(1,7,8,13-18)$. The articles included in this study were published from 2010 to 2018, and the sample size was between 10 and 110 . The present metaanalysis included 323 patients in the Karlsson group and 320 patients in the Broström group. 
Table 1 Characteristic of the included studies

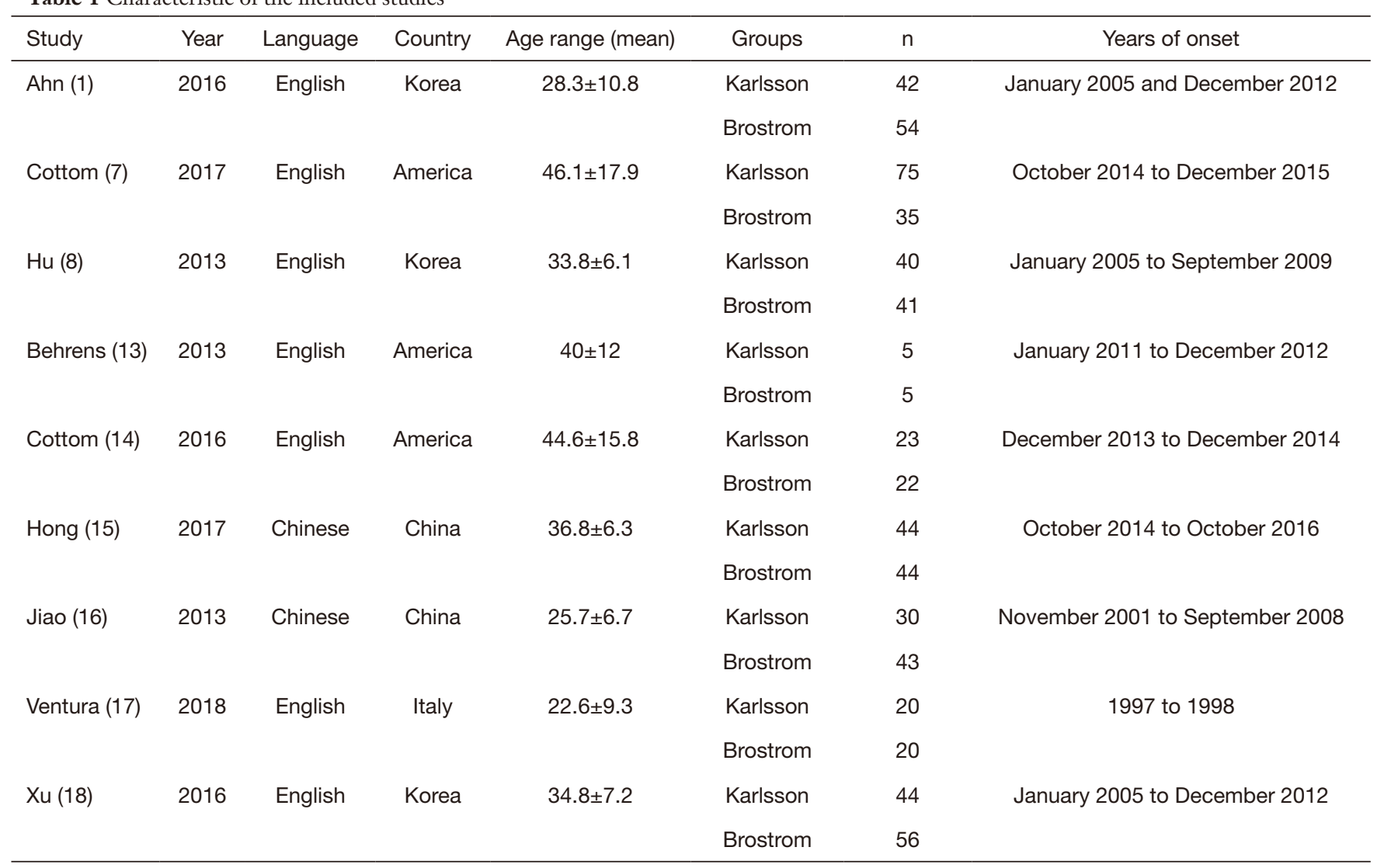

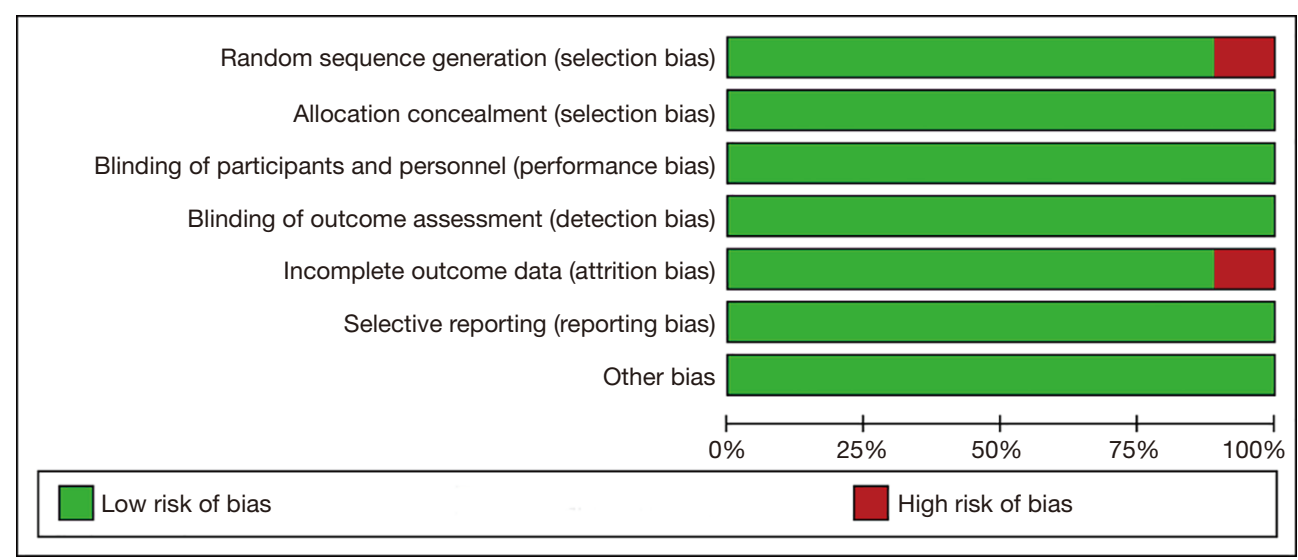

Figure 2 Red and green indicate high-deviation risk and low-deviation risk respectively.

\section{Quality assessment}

Review Manager 5.0 assesses the risk of each study by assessing related deviations. The risk of bias in this study is shown in Figure 2. The investigator found almost no prejudice between the Karlsson and Broström groups.

\section{Results of the meta-analysis}

\section{Meta-analysis of AOFAS scores}

A total of 9 studies comprising 643 patients included American Association of Foot and Ankle Surgery (AOFAS) scores (19). Figure 3 shows a standard map of the AOFAS 


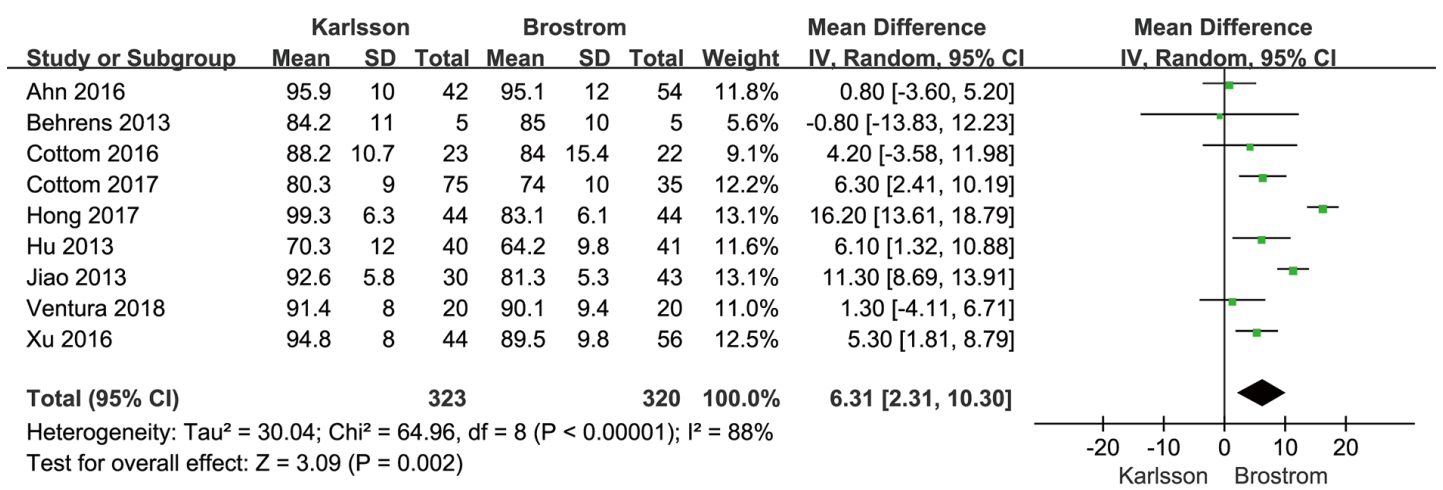

Figure 3 Forest plot for AOFAS scores in the Karlsson and Broström groups. AOFAS, American Orthopedic Foot and Ankle Society.

\begin{tabular}{|c|c|c|c|c|c|c|c|c|c|}
\hline \multirow[b]{2}{*}{ Study or Subgroup } & \multicolumn{3}{|c|}{ Karlsson } & \multicolumn{3}{|c|}{ Brostrom } & \multicolumn{2}{|r|}{ Mean Difference } & \multirow{2}{*}{$\begin{array}{l}\text { Mean Difference } \\
\text { IV. Fixed, } 95 \% \mathrm{CI}\end{array}$} \\
\hline & Mean & SD & Total & Mean & SD & Total & Weight & IV, Fixed, $95 \% \mathrm{Cl}$ & \\
\hline Ahn 2016 & 6.6 & 1.3 & 42 & 6 & 1.5 & 54 & $17.5 \%$ & $0.60[0.04,1.16]$ & \\
\hline Behrens 2013 & 6.8 & 1.5 & 5 & 5.8 & 1.6 & 5 & $1.5 \%$ & $1.00[-0.92,2.92]$ & \\
\hline Cottom 2016 & 6.6 & 1.4 & 23 & 5.6 & 1.5 & 22 & $7.6 \%$ & $1.00[0.15,1.85]$ & \\
\hline Cottom 2017 & 7.1 & 1.5 & 75 & 6.4 & 1.3 & 35 & $18.3 \%$ & $0.70[0.15,1.25]$ & \\
\hline Hong 2017 & 6.5 & 1.8 & 44 & 5.9 & 1.8 & 44 & $9.7 \%$ & $0.60[-0.15,1.35]$ & \\
\hline Hu 2013 & 7.3 & 1.7 & 40 & 6.7 & 1.9 & 41 & $8.9 \%$ & $0.60[-0.18,1.38]$ & \\
\hline Jiao 2013 & 6.2 & 1.3 & 30 & 5.8 & 1.2 & 43 & $15.9 \%$ & $0.40[-0.19,0.99]$ & \\
\hline Ventura 2018 & 5.5 & 1.5 & 20 & 5.5 & 1.6 & 20 & $6.0 \%$ & $0.00[-0.96,0.96]$ & \\
\hline Xu 2016 & 7.9 & 1.6 & 44 & 6.4 & 1.5 & 56 & $14.6 \%$ & $1.50[0.89,2.11]$ & \\
\hline Total $(95 \% \mathrm{Cl})$ & & & 323 & & & 320 & $100.0 \%$ & $0.72[0.48,0.95]$ & \\
\hline $\begin{array}{l}\text { Heterogeneity: } \mathrm{Chi}^{2}= \\
\text { Test for overall effect: }\end{array}$ & $\begin{array}{l}10.35, d f \\
Z=6.00\end{array}$ & $\begin{array}{l}=8 \\
(P<\end{array}$ & $\begin{array}{l}P=0.2 \\
0.0000\end{array}$ & $\begin{array}{l}\text { 4); }\left.\right|^{2}= \\
\text { 1) }\end{array}$ & $3 \%$ & & & & $\begin{array}{llll}1 & 1 & & 1 \\
-2 & -1 & 0 & 1\end{array}$ \\
\hline
\end{tabular}

Figure 4 Forest plot for Tegner scores in the Karlsson and Broström groups.

scores. The differences between the scores for the Karlsson and Broström groups were statistically significant. The metaanalysis suggested that the difference in the AOFAS scores were as follows: mean deviation $(\mathrm{MD})=6.31,95 \%$ CI: $2.31-$ 10.30, $\mathrm{P}=0.002$; $\mathrm{P}$ for heterogeneity $<0.00001, \mathrm{I}^{2}=58 \%$. The AOFAS scores of patients in the Karlsson group were higher than those of patients in the Broström group.

\section{Meta-analysis of the Tegner scores}

Figure 4 shows a forest plot for a meta-analysis of the Tegner scores (20) for the 643 patients. The results showed that the Tegner scores of patients in the Karlsson group were higher than those in the Broström group ( $M D=0.72,95 \%$ CI: $0.48-$ $0.95, \mathrm{P}=0.24$; $\mathrm{P}$ for heterogeneity $<0.00001, \mathrm{I}^{2}=23 \%$ ).

\section{Meta-analysis of operation times}

This meta-analysis included 643 samples with data on the operation times for patients who underwent surgery to treat CLAI (see Figure 5). The overall results indicated that the operation times of patients in the Broström group were higher than those of patients in the Karlsson group $(\mathrm{MD}=-15.50,95 \% \mathrm{CI}:-19.98--11.02, \mathrm{P}<0.00001$; $\mathrm{P}$ for heterogeneity $\left.<0.00001, \mathrm{I}^{2}=63 \%\right)$.

\section{Meta-analysis of patients' satisfaction levels}

As Figure 6 shows, 9 studies comprising 643 samples were included in the meta-analysis comparing the satisfaction of patients. All these studies found statistically significant differences between the Karlsson and Broström groups in terms of satisfaction. Additionally, the combined results showed that patients in the Karlsson group had higher levels of satisfaction that those in the Broström group $(\mathrm{MD}=0.63,95 \% \mathrm{CI}: 0.47-0.79, \mathrm{P}<0.00001 ; \mathrm{P}$ for heterogeneity $=0.91, \mathrm{I}^{2}=0 \%$ ).

\section{Meta-analysis of surgery complications}

All relevant articles (comprising 643 samples) were evaluated to examine surgery complications. As Figure 7 


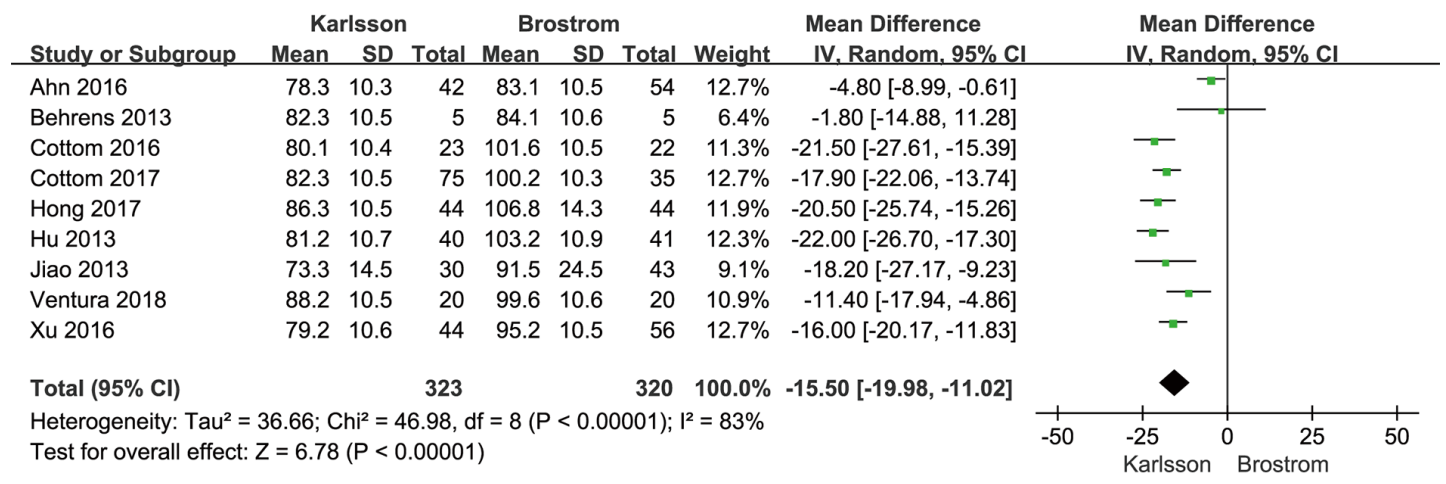

Figure 5 Forest plot for operation times in the Karlsson and Broström groups.

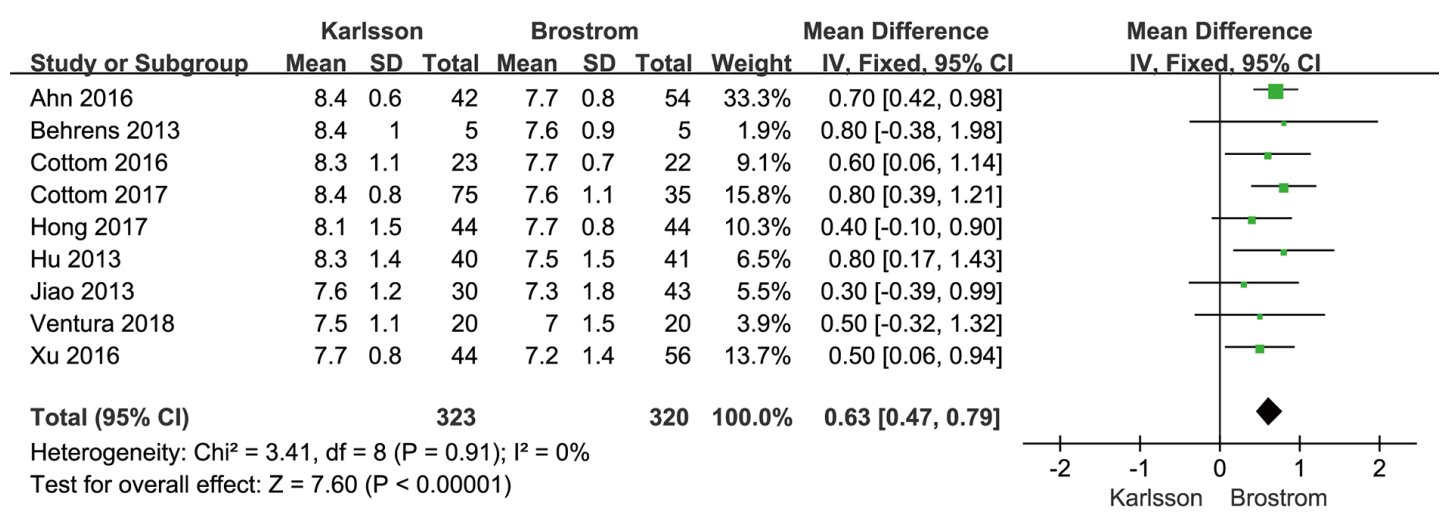

Figure 6 Forest plot for the satisfaction levels of patients in the Karlsson and Broström groups.

shows, no significant difference was observed in relation to surgery complications between the Karlsson and Broström groups $(\mathrm{OR}=1.71,95 \% \mathrm{CI}: 0.79-3.71, \mathrm{P}=0.18$; $\mathrm{P}$ for heterogeneity $\left.=0.99, \mathrm{I}^{2}=0 \%\right)$.

\section{Sensitivity analysis}

As Figure 8 shows, the meta-analysis results revealed that the heterogeneity of the AOFAS scores was high $\left(\mathrm{I}^{2}=88 \%\right)$. The high heterogeneity of the AOFAS scores may be attributed to the results of study by Hong (15). Interestingly, when data from Hong's (15) study were excluded from the metaanalysis, the $\mathrm{I}^{2}$ decreased to $72 \%$.

\section{Bias analysis}

Funnel plots of the AOFAS scores in the Karlsson and Broström groups were created for all the studies (see Figure 9). The results of the funnel plot showed medium symmetry and little publication bias. However, the results of the Begg's test $(\mathrm{z}=0.54, \mathrm{P}=0.423)$ and Egger's test $(\mathrm{T}=-1.21, \mathrm{P}=0.101)$ did not show any publication bias.

\section{Discussion}

The ankle joint is the most commonly injured part of the human motion system. The most vulnerable part is the lateral collateral ligament of the ankle joint (21-23). Reportedly, of patients with ankle joint injuries, $85 \%$ have an injury of the lateral collateral ligament.

For most patients with ankle sprains, satisfactory results can be obtained after conservative treatment and some patients can be cured; however, about $20 \%$ of the patients' injuries gradually worsen, and up to $40 \%$ of the patients eventually present with CLAI (24-26). The typical symptoms of CLAI include ankle pain while walking, repeated ankle sprains, weakness of lower limbs, and a fear of walking on uneven ground. 


\begin{tabular}{|c|c|c|c|c|c|c|c|c|c|c|}
\hline Study or Subgroup & $\begin{array}{l}\text { Karlssc } \\
\text { Events }\end{array}$ & $\begin{array}{l}\text { on } \\
\text { Total }\end{array}$ & $\begin{array}{l}\text { Brostro } \\
\text { Events }\end{array}$ & $\begin{array}{l}\text { Total } \\
\text { Total }\end{array}$ & Weight & $\begin{array}{c}\text { Odds Ratio } \\
\text { M-H, Fixed, } 95 \% \mathrm{Cl}\end{array}$ & & $\begin{array}{r}\text { Odds } \\
\text { M-H. Fixe }\end{array}$ & $\begin{array}{l}\text { Ratio } \\
\text { ed. } 95 \% \mathrm{Cl}\end{array}$ & \\
\hline Ahn 2016 & 2 & 42 & 2 & 54 & $16.6 \%$ & $1.30[0.18,9.63]$ & & & & \\
\hline Behrens 2013 & 0 & 5 & 0 & 5 & & Not estimable & & & & \\
\hline Cottom 2016 & 2 & 23 & 2 & 22 & $18.5 \%$ & $0.95[0.12,7.42]$ & & & & \\
\hline Cottom 2017 & 3 & 75 & 1 & 35 & $13.0 \%$ & $1.42[0.14,14.12]$ & & & & \\
\hline Hong 2017 & 3 & 44 & 1 & 44 & $9.3 \%$ & $3.15[0.31,31.48]$ & & & & \\
\hline Hu 2013 & 2 & 40 & 1 & 41 & $9.3 \%$ & $2.11[0.18,24.18]$ & & & & \\
\hline Jiao 2013 & 2 & 30 & 1 & 43 & $7.6 \%$ & $3.00[0.26,34.68]$ & & & & \\
\hline Ventura 2018 & 1 & 20 & 1 & 20 & $9.4 \%$ & $1.00[0.06,17.18]$ & & & & \\
\hline Xu 2016 & 3 & 44 & 2 & 56 & $16.3 \%$ & $1.98[0.32,12.37]$ & & & & \\
\hline Total $(95 \% \mathrm{Cl})$ & & 323 & & 320 & $100.0 \%$ & $1.71[0.79,3.71]$ & & & & \\
\hline Total events & 18 & & 11 & & & & & & & \\
\hline \multicolumn{7}{|c|}{$\begin{array}{l}\text { Heterogeneity: } \mathrm{Chi}^{2}=1.07, \mathrm{df}=7(P=0.99) ; I^{2}=0 \% \\
\text { Test for overall effect: } Z=1.35(P=0.18)\end{array}$} & .001 & $\begin{array}{c}0.1 \\
\text { Karlsson }\end{array}$ & $\begin{array}{c}10 \\
\text { Brostrom }\end{array}$ & 1000 \\
\hline
\end{tabular}

Figure 7 Forest plot for complications in the Karlsson and Broström groups.

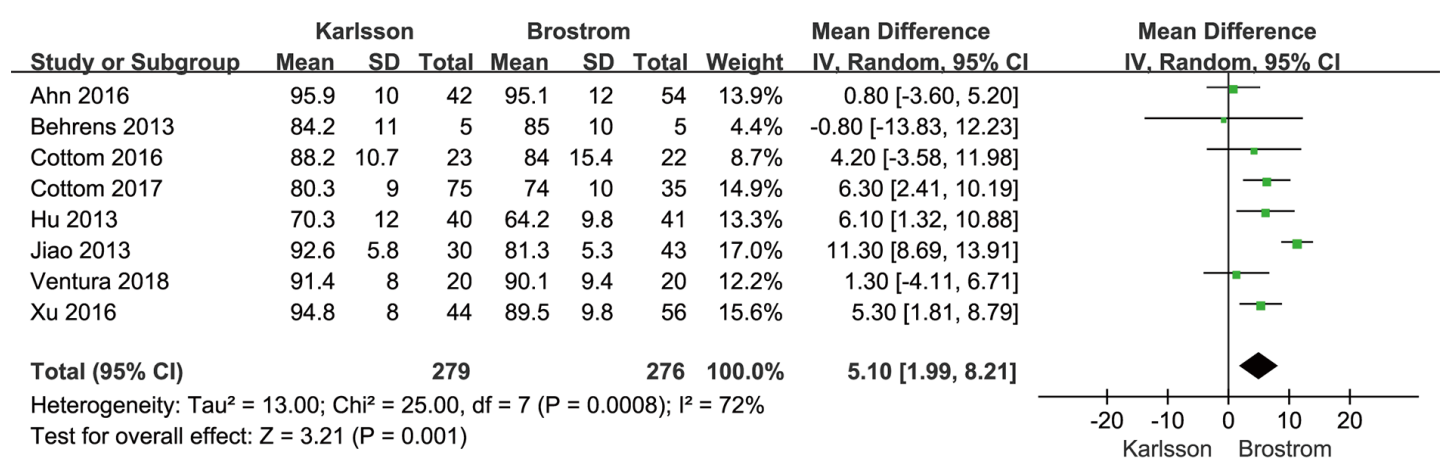

Figure 8 Forest plot for the sensitivity analysis of the AOFAS scores in the Karlsson and Broström groups. AOFAS, American Orthopedic Foot and Ankle Society.

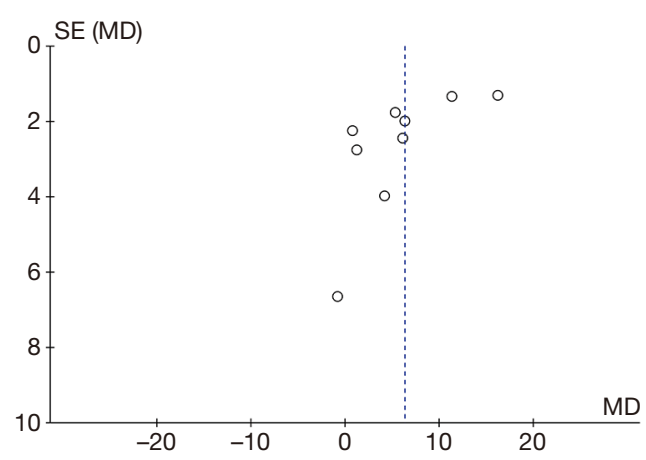

Figure 9 Begg's funnel plot of publication bias.

In 1988, Karlsson reported that the distal fibula could be repaired by an imbricate suture using a multi-drilling technique. In one study, a total of 152 patients were followed up for 2-10 years after surgery, and a rate of $87 \%$ in recovery was reported (27). In 1964, Broström reported 60 cases of direct ligament repair. The method of tightening and suturing the joint capsule is suitable for patients with CLAI (28-30). However, the incidence of complications is low and the clinical effect is satisfactory, albeit some patients' ankles will remain unstable.

In this study, the difference in the AOFAS scores between the Karlsson and Broström groups was statistically significant. Further, the Tegner scores and patients' level of satisfaction were also significant variables. These results showed that the clinical effects for patients in the Karlsson group were better than those for patients the Broström group, which supports the results of Cho et al.'s study on CLAI. The AOFAS scores of patients in the modified Karlsson operation group were significantly lower than those of patients in the modified Broström group before the operation. After treatment, the AOFAS scores of patients in 
the modified Karlsson operation group increased, but not to a level that was significantly different from those of patients in the modified Broström group. Further, the improved scores were significantly higher for patients in the Karlsson group than those for patients in the Broström group, which indicates that the Karlsson treatment effect might be slightly better than the Broström treatment effect (5).

Operation times for patients in the Broström group were not significantly higher than those of patients in the Karlsson group. Chun et al. contended that the modified Karlsson procedure is similar and easier than the modified Broström procedure (6). The modified Karlsson's procedure alters the way in which the bone passage is made when reconstructing the original operation termination point to suture and fix the broken end of the ligament with a suture anchor at the ligament termination point, which reduces the time needed for making the bone passage and shortens the operation time compared to the modified Broström's method.

It should be noted that the present study had some limitations. To address these issues, the analysis indicator in both the Karlsson and Broström groups should be analyzed and evaluated in the future. Further, additional updated articles from other countries should be investigated in further research.

Taken together, the results showed that the Karlsson procedure is better than the Broström procedure in treating CLAI. These results support those of previous studies. In the present study, the meta-analyses had low heterogeneities, and according to the funnel plots and Begg's test, no publication bias was observed, which provides further evidence in support of these results.

\section{Acknowledgments}

Funding: Supported by "Health Commission of Hubei Province scientific research project" (No. WJ2019F152, WJ2021F114).

\section{Footnote}

Reporting Checklist: The authors have completed the PRISMA reporting checklist. Available at https://dx.doi. org/10.21037/apm-21-963

Conflicts of Interest: All authors have completed the ICMJE uniform disclosure form (available at https://dx.doi. org/10.21037/apm-21-963). The authors have no conflicts of interest to declare.
Ethical Statement: The authors are accountable for all aspects of the work in ensuring that questions related to the accuracy or integrity of any part of the work are appropriately investigated and resolved.

Open Access Statement: This is an Open Access article distributed in accordance with the Creative Commons Attribution-NonCommercial-NoDerivs 4.0 International License (CC BY-NC-ND 4.0), which permits the noncommercial replication and distribution of the article with the strict proviso that no changes or edits are made and the original work is properly cited (including links to both the formal publication through the relevant DOI and the license). See: https://creativecommons.org/licenses/by-nc-nd/4.0/.

\section{References}

1. Ahn HW, Lee KB. Comparison of the Modified Broström Procedure for Chronic Lateral Ankle Instability With and Without Subfibular Ossicle. Am J Sports Med 2016;44:3158-64.

2. Bell SJ, Walthour CS, Provencher MT, et al. Chronic Lateral Ankle Instability: The Brostrm Procedure. Oper Techn Sport Med 2005;13:176-82.

3. Brodsky AR, O'Malley MJ, Bohne WH, et al. An analysis of outcome measures following the Broström-Gould procedure for chronic lateral ankle instability. Foot Ankle Int 2005;26:816-9.

4. Buerer Y, Winkler M, Burn A, et al. Evaluation of a modified Broström-Gould procedure for treatment of chronic lateral ankle instability: A retrospective study with critical analysis of outcome scoring. Foot Ankle Surg 2013;19:36-41.

5. Cho BK, Kim YM, Shon HC, et al. A ligament reattachment technique for high-demand athletes with chronic ankle instability. J Foot Ankle Surg 2015;54:7-12.

6. Chun TH, Park YS, Sung KS. The effect of ossicle resection in the lateral ligament repair for treatment of chronic lateral ankle instability. Foot Ankle Int 2013;34:1128-33.

7. Cottom JM, Baker J, Plemmons BS. Analysis of Two Different Arthroscopic Broström Repair Constructs for Treatment of Chronic Lateral Ankle Instability in 110 Patients: A Retrospective Cohort Study. J Foot Ankle Surg 2018;57:31-7.

8. Hu CY, Lee KB, Song EK, et al. Comparison of bone tunnel and suture anchor techniques in the modified Broström procedure for chronic lateral ankle instability. 
Am J Sports Med 2013;41:1877-84.

9. Dierckman BD, Ferkel RD. Anatomic Reconstruction With a Semitendinosus Allograft for Chronic Lateral Ankle Instability. Am J Sports Med 2015;43:1941-50.

10. Eechaute C, Vaes $\mathrm{P}$, Aerschot LV, et al. The clinimetric qualities of patient-assessed instruments for measuring chronic ankle instability: A systematic review. BMC Musculoskelet Disord 2007;8:6.

11. Hennrikus WL, Mapes RC, Lyons PM, et al. Outcomes of the Chrisman-Snook and modified-Broström procedures for chronic lateral ankle instability. A prospective, randomized comparison. Am J Sports Med 1996;24:400-4.

12. Karlsson J, Bergsten T, Lansinger O, et al. Surgical treatment of chronic lateral instability of the ankle joint. A new procedure. Am J Sports Med 1989;17:268-73; discussion 273-4.

13. Behrens SB, Drakos M, Lee BJ, et al. Biomechanical analysis of Brostrom versus Brostrom-Gould lateral ankle instability repairs. Foot Ankle Int 2013;34:587-92.

14. Cottom JM, Baker JS, Richardson PE. The "All-Inside" Arthroscopic Broström Procedure With Additional Suture Anchor Augmentation: A Prospective Study of 45 Consecutive Patients. J Foot Ankle Surg 2016;55:1223-8.

15. Hong S, Lin H, Yang C, et al. Comparison of the Effect Between Anatomical Reconstruction and Modified Brostrom Suture Repair for Chronic Lateral Ankle Instability. China Health Standard Management 2017;31:81-90.

16. Jiao C, Hu YL, Guo QW, et al. Repairing ankle lateral collateral ligament chronic injury using different methods: A stability assessment. Chinese Journal of Tissue Engineering Research 2013;2:65-72.

17. Ventura A, Legnani C, Corradini C, et al. Lateral ligament reconstruction and augmented direct anatomical repair restore ligament laxity in patients suffering from chronic ankle instability up to 15 years from surgery. Knee Surg Sports Traumatol Arthrosc 2020;28:202-7.

18. Xu HX, Choi MS, Kim MS, et al. Gender Differences in Outcome After Modified Broström Procedure for Chronic Lateral Ankle Instability. Foot Ankle Int 2016;37:64-9.

19. Kitaoka HB, Alexander IJ, Adelaar RS, et al. Clinical rating systems for the ankle-hindfoot, midfoot, hallux, and lesser toes. Foot Ankle Int 1994;15:349-53.

20. Caplan N, Kader DF. Rating Systems in the Evaluation of Knee Ligament Injuries. Classic Papers in Orthopaedics 2014;12:362-75.

21. So E, Preston N, Holmes T. Intermediate- to LongTerm Longevity and Incidence of Revision of the Modified Broström-Gould Procedure for Lateral Ankle Ligament Repair: A Systematic Review. J Foot Ankle Surg 2017;56:1076-80.

22. Xu HX, Choi MS, Kim MS, et al. Gender Differences in Outcome After Modified Broström Procedure for Chronic Lateral Ankle Instability. Foot Ankle Int 2016;37:64-9.

23. Yang Q, Zhou Y, Xu Y. Arthroscopic debridement of anterior ankle impingement in patients with chronic lateral ankle instability. BMC Musculoskelet Disord 2018;19:239.

24. Miyamoto W, Takao M, Yamada K, et al. Accelerated Versus Traditional Rehabilitation After Anterior Talofibular Ligament Reconstruction for Chronic Lateral Instability of the Ankle in Athletes. Am J Sports Med 2014;42:1441-7.

25. Murphy GA. The modified Broström techniquefor reconstruction of chronic lateral ankle instability. Oper Techn Sport Med 1999;23:672-9.

26. Russo A, Giacchè P, Marcantoni E, et al. Treatment of chronic lateral ankle instability using the Broström-Gould procedure in athletes: long-term results. Joints 2016;4:94-7.

27. Maffulli N, Ferran NA. Management of acute and chronic ankle instability. J Am Acad Orthop Surg 2008;16:608-15.

28. Karlsson J, Lansinger O. Lateral instability of the ankle joint. Clin Orthop Relat Res 1992;253-61.

29. Krips R, van Dijk CN, Halasi PT, et al. Long-term outcome of anatomical reconstruction versus tenodesis for the treatment of chronic anterolateral instability of the ankle joint: a multicenter study. Foot Ankle Int 2001;22:415-21.

30. Li X, Lin TJ, Busconi BD. Treatment of chronic lateral ankle instability: a modified Broström technique using three suture anchors. J Orthop Surg Res 2009;4:41.

(English Language Editor: L. Huleatt)
Cite this article as: Deng X, Zou M, Zhu H, Zuo C, Li K, Qian L. A comparison of the modified Broström procedure and modified Karlsson procedure in treating chronic lateral ankle instability: a systematic review and meta-analysis. Ann Palliat Med 2021;10(7):7534-7542. doi: 10.21037/apm-21-963 